\title{
COMPLEX STRESS FUNCTIONS AND PLASTIC ZONE SIZES FOR NOTCH CRACKS SUBJECTED TO VARIOUS LOADING CONDITIONS
}

\author{
Hong Youshi†, K. J. MiLler and M. W. BRown \\ Department of Mechanical and Process Engineering, University of Sheffield, Mappin Street, \\ Sheffield S1 3JD, England
}

\begin{abstract}
In this paper, the conformal mapping method is used to solve the plane problem of an infinite plate containing a central lip-shaped notch subjected to biaxial loading at a remote boundary or a surface uniform pressure on the notch. The stress intensity factors $K_{\mathrm{I}}$ and $K_{\mathrm{II}}$ are obtained by the derived complex stress functions. The simple analytical expressions can be applied to the situation of cracks originating from a circular or an elliptical notch. The plastic zone sizes for such notch cracks are subsequently evaluated in light of the Dugdale strip yield concept. The results are consistent with available numerical data.
\end{abstract}

\section{INTRODUCTION}

The stress distribution ahead of a notch and the stress intensity factor at the tip of a crack in a notch field are vital factors in fatigue investigations and failure analyses, since cracks nearly always initiate from a notch root, prior to final fracture. Some papers [1-7] have dealt with this problem using different approaches based on fracture mechanics from the time this discipline was introduced into engineering design, research and lifetime prediction. However, most solutions are given as numerical results.

The engineering significance of stresses and stress intensity factors in a notch field has also attracted scientific efforts on deriving analytical or empirical expressions describing these parameters in relation to the notch-crack size and loading conditions. After examining a variety of experimental data of central and edge circular or elliptical notches, Smith and Miller [8] proposed an approximation for the stress intensity factor $K$ of a crack of length $l$ from a notch root under a remote uniaxial tensile stress $\sigma$

$$
K=\left[1+7.69 \sqrt{\frac{D}{\rho}}\right]^{1 / 2} \sigma \sqrt{\pi l} .
$$

The limitation of this is that the formula conforms well to the theoretical result only when $l<0.1 \sqrt{D \rho}$, where $D$ is the depth of a notch and $\rho$ is notch root radius.

Among the theoretical procedures for evaluating the stress distribution ahead of a notch, the complex stress function is a method initially developed by Muskhelishvili[9], which is one available for solving problems for various notch shapes via conformal mapping. Later, the theory was further advanced by Paris and Sih[10] who established a relationship between stress intensity factors and a complex stress function.

In this paper, we use the complex stress function method to solve the problem of infinite plates containing a central lip-shaped notch subjected to biaxial loading at a remote boundary or for a

†Visiting Royal Fellow from the Institute of Mechanics, Chinese Academy of Sciences, Beijing 100080, P.R. China. 

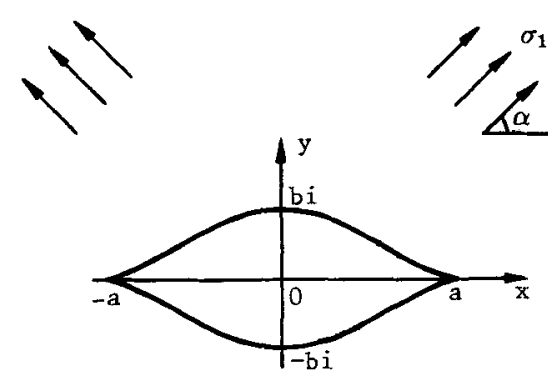

(a)



(b)

Fig. 1. Infinite plate containing a central lip-shaped notch under (a) remote biaxial loading. (b) Uniform pressure on the notch boundary profile.

uniform surface pressure on the notch profile, this being followed by an evaluation of the stress intensity factor at the notch crack tip. The analytical solutions are found to be suitable for configurations of cracks originating from a circular or an elliptical notch. Moreover, the plastic zone sizes for such notch cracks can be determined via the Dugdale strip yield concept [11].

\section{THEORETICAL CONSIDERATIONS}

\section{Conformal mapping}

Consider an infinite thin plate with a central lip-shaped notch subjected to remote boundary biaxial loading or a uniform pressure on notch profile itself (Fig. 1). The conformal mapping method is used to derive the complex stress functions of the problem. First, the following formula is adopted to transform a lip-shape profile on the $z$-plane to an ellipse on the $t$-plane [Fig. 2(a-b)].

$$
z=\frac{a}{2}\left(t+\frac{1}{t}\right)
$$

where $a$ is the half length of the lip-shape profile, and the half width of it, $b$, is assumed always be unity. Secondly, we use

$$
t=r\left(\zeta+\frac{m}{\zeta}\right)
$$

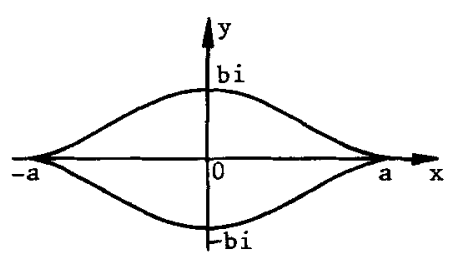

(a) $z$-plane

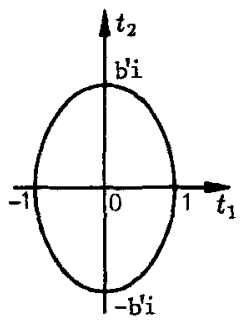

(b) $t$-plane

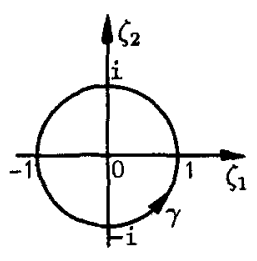

(c) $\zeta$-plane

Fig. 2. Mapping a lip-shape profile onto a circle. 
to transform an ellipse on the $t$-plane to a unit circle on the $\zeta$-plane [Fig. 2(b-c)], and thus one can show

$$
r(1+m)=1 .
$$

Combining the above two transformations, we obtain

$$
z=\omega(\zeta)=R\left[\zeta+\frac{m}{\zeta}+\frac{\zeta}{r^{2}\left(\zeta^{2}+m\right)}\right]
$$

where

$$
R=\frac{a}{2} r
$$

Equation (4) uniquely maps the outer region of a lip-shape profile on the $z$-plane onto the exterior of a unit circle on the $\zeta$-plane. The parameters $r$ and $m$ are dimensionless and can be found to be

$$
r=\frac{b}{2 a}\left[1+\frac{a}{b}+\sqrt{1+\left(\frac{a}{b}\right)^{2}}\right]
$$

and

$$
m=\frac{a}{b}-\sqrt{1+\left(\frac{a}{b}\right)^{2}}
$$

Note that, since $a / b \geqslant 0$

$$
-1 \leqslant m \leqslant 0 .
$$

Let $\gamma$ be the circumference of the unit circle of the $\zeta$-plane, and so equation (4) has the form

$$
\omega(\eta)=\frac{a}{2} r\left[\eta+\frac{m}{\eta}+\frac{\eta}{r^{2}\left(\eta^{2}+m\right)}\right]
$$

where $\eta=\mathrm{e}^{i \theta}$. Noting that $z=x+i y$, we obtain, from equation (9)

$$
\left.\begin{array}{l}
x=\frac{a}{2}\left[1+\frac{(1+m)^{2}}{1+m^{2}+2 m \cos 2 \theta}\right] \cos \theta \\
y=\frac{a}{2} \cdot \frac{(1-m)}{(1+m)}\left[1-\frac{(1+m)^{2}}{1+m^{2}+2 m \cos 2 \theta}\right] \sin \theta .
\end{array}\right\}
$$

The above equation describes the contour of a lip-shaped notch on the $z$-plane (Fig. 3).

\section{Basic equations}

According to the theory developed by Muskhelishvili[9], the complex stress functions for the general case on the $\zeta$-plane are

$$
\begin{aligned}
& \phi(\zeta)=\Gamma R \zeta-\frac{X+i Y}{2 \pi(1+\kappa)} \ln \zeta+\phi_{0}(\zeta) \\
& \psi(\zeta)=\Gamma_{1} R \zeta+\frac{\kappa(X-i Y)}{2 \pi(1+\kappa)} \ln \zeta+\psi_{0}(\zeta)
\end{aligned}
$$




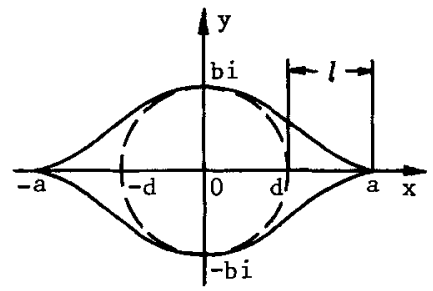

(a)

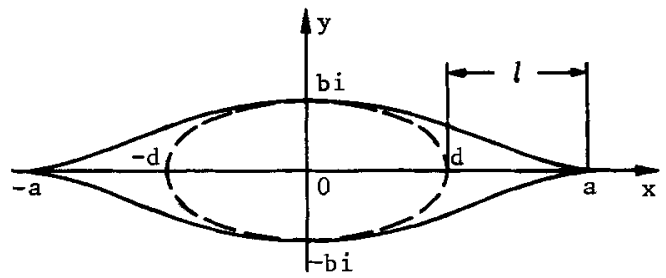

(b)

Fig. 3. Solid curves showing the contours of two lip-shaped notches. (a) $a / b=2.0, m=-0.2361$ and $r=1.309$; enclosing a circle of $d=b$. (b) $a / b=4.0, m=-0.1231$ and $r=1.140$; enclosing an ellipse of $d=2 b$.

where $\phi_{0}(\zeta)$ and $\psi_{0}(\zeta)$ are holomorphic for $|\zeta|>1$, and

$$
\phi_{0}(\infty)=\psi_{0}(\infty)=0
$$

while on $\gamma$

$$
\begin{aligned}
& \phi(\eta)=\Gamma R \eta-\frac{X+i Y}{2 \pi(1+\kappa)} \ln \eta+\phi_{0}(\eta) \\
& \psi(\eta)=\Gamma_{1} R \eta+\frac{\kappa(X-i Y)}{2 \pi(1+\kappa)} \ln \eta+\psi_{0}(\eta)
\end{aligned}
$$

where $X$ and $Y$ are the resultant forces on the notch boundary, and $\Gamma$ and $\Gamma_{1}$ are constants depending on the remote loading conditions. Note that, for plane stress

$$
\kappa=\frac{3-v}{1+v}
$$

where $v$ is Poisson's ratio.

On the other hand, the boundary conditions for the general case on the $\zeta$-plane are such that

$$
\phi(\eta)+\frac{\omega(\eta)}{\overline{\omega^{\prime}(\eta)}} \overline{\phi^{\prime}(\eta)}+\overline{\psi(\eta)}=f
$$

where the prime notation denotes differentiation with respect to $\eta$ and the bar denotes the complex conjugate. In the above equation

$$
f=i \int_{0}^{s}\left(X_{n}+i Y_{n}\right) \mathrm{d} s
$$

where $X_{n}$ and $Y_{n}$ are the resultant amounts of the positive normal stress on the element $\mathrm{d} s$ of the boundary. Substituting equations (14), (15) and their conjugates into (16), one obtains

$$
\phi_{0}(\eta)+\frac{\omega(\eta)}{\overline{\omega^{\prime}(\eta)}} \overline{\phi_{0}^{\prime}(\eta)}+\overline{\psi_{0}(\eta)}=f_{0}
$$

and

$$
\overline{\phi_{0}(\eta)}+\frac{\overline{\omega(\eta)}}{\omega^{\prime}(\eta)} \phi_{0}^{\prime}(\eta)+\psi_{0}(\eta)=\bar{f}_{0}
$$


where

$$
f_{0}=f-\Gamma R \eta+\frac{X+i Y}{2 \pi} \ln \eta-\frac{\omega(\eta)}{\overline{\omega^{\prime}(\eta)}}\left[\Gamma R-\frac{X-i Y}{2 \pi(1+\kappa)} \eta\right]-\overline{\Gamma_{1}} R \frac{1}{\eta}
$$

and

$$
\overline{f_{0}}=\bar{f}-\bar{\Gamma} R \frac{1}{\eta}-\frac{X-i Y}{2 \pi} \ln \eta-\frac{\overline{\omega(\eta)}}{\omega^{\prime}(\eta)}\left[\bar{\Gamma} R-\frac{X+i Y}{2 \pi(1+\kappa)} \cdot \frac{1}{\eta}\right]-\Gamma_{1} R \eta
$$

\section{SOME SOLUTIONS}

\section{Case 1: remote biaxial loading}

Boundary conditions. When a plate contains a central lip-shaped notch and is subjected to remote biaxial loading [Fig. 1(a)], the corresponding boundary conditions are

$$
\begin{aligned}
& \Gamma=\bar{\Gamma}=\frac{1}{4}\left(\sigma_{1}+\sigma_{2}\right) \\
& \Gamma_{1}=-\frac{1}{2}\left(\sigma_{1}-\sigma_{2}\right) \mathrm{e}^{-2 i x}
\end{aligned}
$$

where the first equation is written such that no rotation at infinity is assumed, $\sigma_{1}$ and $\sigma_{2}$ are the remote principal stresses due to external loading, and $\alpha$ is the angle made by the direction of $\sigma_{1}$ with reference to the axis $0 x$; and

$$
\begin{aligned}
f & =0 \\
X+i Y & =0 .
\end{aligned}
$$

Therefore, equations (20) and (21) become

$$
f_{0}=-\frac{R}{4}\left(\sigma_{1}+\sigma_{2}\right)\left[\eta+\frac{\omega(\eta)}{\overline{\omega^{\prime}(\eta)}}\right]+\frac{R}{2}\left(\sigma_{1}-\sigma_{2}\right) \frac{1}{\eta} \mathrm{e}^{2 i \alpha}
$$

and

$$
\overline{f_{0}}=-\frac{R}{4}\left(\sigma_{1}+\sigma_{2}\right)\left[\frac{1}{\eta}+\overline{\frac{\omega(\eta)}{\omega^{\prime}(\eta)}}\right]+\frac{R}{2}\left(\sigma_{1}-\sigma_{2}\right) \eta \mathrm{e}^{-2 i \alpha}
$$

where

$$
\frac{\omega(\eta)}{\overline{\omega^{\prime}(\eta)}}=\frac{\left[r^{2}\left(\eta^{2}+m\right)^{2}+\eta^{2}\right]\left(1+m \eta^{2}\right)^{2}}{r^{2} \eta\left(m+\eta^{2}\right)\left(1-\eta^{2}\right)\left(1-m \eta^{2}\right)\left(1-m^{2} \eta^{2}\right)}
$$

and

$$
\frac{\overline{\omega(\eta)}}{\omega^{\prime}(\eta)}=\frac{\eta\left[r^{2}\left(1+m \eta^{2}\right)^{2}+\eta^{2}\right]\left(\eta^{2}+m\right)^{2}}{r^{2}\left(1+m \eta^{2}\right)\left(\eta^{2}-1\right)\left(\eta^{2}-m\right)\left(\eta^{2}-m^{2}\right)}
$$

which are deduced from the mapping function [equation (9)]. 
Complex stress functions. Since $\phi_{0}(\zeta)$ and $\psi_{0}(\zeta)$ are holomorphic outside $\gamma$, and $\overline{\phi_{0}(\zeta)}$ and $\overline{\psi_{0}(\zeta)}$ are holomorphic inside $\gamma$, we have, according to the Cauchy integral theorem

$$
\begin{array}{ll}
\frac{1}{2 \pi i} \int_{\gamma} \frac{\phi_{0}(\eta)}{\eta-\zeta} \mathrm{d} \eta=-\phi_{0}(\zeta) & |\zeta|>1 \\
\frac{1}{2 \pi i} \int_{\gamma} \frac{\overline{\phi_{0}(\eta)}}{\eta-\zeta} \mathrm{d} \eta=0 & |\zeta|>1 \\
\frac{1}{2 \pi i} \int_{\gamma} \frac{\psi_{0}(\eta)}{\eta-\zeta} \mathrm{d} \eta=-\psi_{0}(\zeta) & |\zeta|>1 \\
\frac{1}{2 \pi i} \int_{\gamma} \frac{\overline{\psi_{0}(\eta)}}{\eta-\zeta} \mathrm{d} \eta=0 & |\zeta|>1 .
\end{array}
$$

Then, equations (18) and (19) can be written as

$$
\phi_{0}(\zeta)=-\frac{1}{2 \pi i} \int_{\gamma} \frac{f_{0} \mathrm{~d} \eta}{\eta-\zeta}+\frac{1}{2 \pi i} \int_{\gamma} \frac{\omega(\eta)}{\overline{\omega^{\prime}(\eta)}} \overline{\phi_{0}^{\prime}(\eta)} \frac{\mathrm{d} \eta}{\eta-\zeta}
$$

and

$$
\psi_{0}(\zeta)=-\frac{1}{2 \pi i} \int_{\gamma} \frac{\overline{f_{0}} \mathrm{~d} \eta}{\eta-\zeta}+\frac{1}{2 \pi i} \int_{\zeta} \frac{\overline{\omega(\eta)}}{\omega^{\prime}(\eta)} \phi_{0}^{\prime}(\eta) \frac{\mathrm{d} \eta}{\eta-\zeta}
$$

where $\left[\omega(\eta) \sqrt{\omega^{\prime}(\eta)}\right] \overline{\phi_{0}^{\prime}(\eta)}$ and $\left[\overline{\omega(\eta)} / \omega^{\prime}(\eta)\right] \phi_{0}^{\prime}(\eta)$ are respectively the boundary values of $\left[\omega(\zeta) / \overline{\omega^{\prime}(\zeta)}\right] \overline{\phi_{0}^{\prime}}(1 / \zeta)$ and $\left[\overline{\omega(\zeta)} / \omega^{\prime}(\zeta)\right] \phi_{0}^{\prime}(\zeta)$ with

$$
\frac{\omega(\zeta)}{\overline{\omega^{\prime}(\zeta)}}=\frac{\left[r^{2}\left(\zeta^{2}+m\right)^{2}+\zeta^{2}\right]\left(1+m \zeta^{2}\right)^{2}}{r^{2} \zeta\left(m+\zeta^{2}\right)\left(1-\zeta^{2}\right)\left(1-m \zeta^{2}\right)\left(1-m^{2} \zeta^{2}\right)}
$$

and

$$
\frac{\overline{\omega(\zeta)}}{\omega^{\prime}(\zeta)}=\frac{\zeta\left[r^{2}\left(1+m \zeta^{2}\right)^{2}+\zeta^{2}\right]\left(\zeta^{2}+m\right)^{2}}{r^{2}\left(1+m \zeta^{2}\right)\left(\zeta^{2}-1\right)\left(\zeta^{2}-m\right)\left(\zeta^{2}-m^{2}\right)}
$$

Note also that

$$
\begin{array}{ll}
\phi_{0}(\zeta)=\frac{c_{1}}{\zeta}+\frac{c_{2}}{\zeta^{2}}+\cdots & |\zeta|>1 \\
\phi_{0}^{\prime}(\zeta)=-\frac{c_{1}}{\zeta^{2}}-\frac{2 c_{2}}{\zeta^{3}}-\cdots & |\zeta|>1
\end{array}
$$

and

$$
\overline{\phi_{0}^{\prime}}\left(\frac{1}{\zeta}\right)=-\overline{c_{1}} \zeta^{2}-2 \overline{c_{2}} \zeta^{3}-\cdots|\zeta|<1
$$

We find that $\left[\omega(\zeta) / \overline{\omega^{\prime}(\overline{\zeta)})} \overline{\phi_{0}^{\prime}}(1 / \zeta)\right.$ is holomorphic inside $\gamma$, except at $\zeta= \pm i \sqrt{m}$; and $\left[\overline{\omega(\zeta)} / \omega^{\prime}(\zeta)\right]$ $\phi_{0}^{\prime}(\zeta)$ is holomorphic outside $\gamma$, except at $\zeta= \pm i / \sqrt{m}$. So that, according to the Cauchy integral 
theorem (for $|\zeta|>1)$

$$
\begin{aligned}
\frac{1}{2 \pi i} \int_{\gamma} \frac{\omega(\eta)}{\overline{\omega^{\prime}(\eta)}} \overline{\phi_{0}^{\prime}(\eta)} \frac{\mathrm{d} \eta}{\eta-\zeta} & =\frac{1}{2 \pi i} \int_{\gamma} \frac{\omega(\eta)}{\overline{\omega^{\prime}(\eta)}} \overline{\phi_{0}^{\prime}}\left(\frac{1}{\eta}\right) \frac{\mathrm{d} \eta}{\eta-\zeta} \\
& =-G_{1}(\zeta)-G_{2}(\zeta)=-\frac{M_{0}}{2}\left[\frac{\overline{\phi_{0}^{\prime}\left(\frac{-i}{\sqrt{m}}\right)}}{\zeta-i \sqrt{m}}+\frac{\overline{\phi_{0}^{\prime}}\left(\frac{i}{\zeta \sqrt{m}}\right)}{\zeta+i \sqrt{m}}\right]
\end{aligned}
$$

where $G_{1}(\zeta)$ and $G_{2}(\zeta)$ are respectively the principal parts of $\frac{\omega(\zeta)}{\overline{\omega^{\prime}(\zeta)}} \overline{\phi_{0}^{\prime}(\zeta)}$ for $\zeta= \pm i \sqrt{m}$; and

$$
\begin{aligned}
\frac{1}{2 \pi i} \int_{\gamma} \frac{\overline{\omega(\eta)}}{\omega^{\prime}(\eta)} \phi_{0}^{\prime}(\eta) \frac{\mathrm{d} \eta}{\eta-\zeta} & =-\frac{\overline{\omega(\zeta)}}{\omega^{\prime}(\zeta)} \phi_{0}^{\prime}(\zeta)+H_{1}(\zeta)+H_{2}(\zeta) \\
& =-\frac{\overline{\omega(\zeta)}}{\omega^{\prime}(\zeta)} \phi_{0}^{\prime}(\zeta)+\frac{M_{0}}{2 m}\left[\frac{\phi_{0}^{\prime}\left(\frac{i}{\sqrt{m}}\right)}{\left.\zeta-\frac{i}{\sqrt{m}}+\frac{\phi_{0}^{\prime}\left(\frac{-i}{\sqrt{m}}\right)}{\zeta+\frac{i}{\sqrt{m}}}\right]}\right.
\end{aligned}
$$

where $H_{1}(\zeta)$ and $H_{2}(\zeta)$ are respectively the principal parts of $\left[\overline{\omega(\zeta)} / \omega^{\prime}(\zeta)\right] \phi_{0}^{\prime}(\zeta)$ for $\zeta= \pm i / \sqrt{m}$. In equations (40) and (41), the constant

$$
M_{0}=\frac{\left(1-m^{2}\right)^{2}}{\left(1+m^{2}\right)\left(1-m+m^{2}\right)} .
$$

In order to solve the first term in the right hand side of equations (33) and (34), we again apply the Cauchy integral theorem (for $|\zeta|>1$ )

$$
\begin{aligned}
\frac{1}{2 \pi i} \int_{\gamma} \frac{\eta \mathrm{d} \eta}{\eta-\zeta} & =0 \\
\frac{1}{2 \pi i} \int_{\gamma} \frac{\mathrm{d} \eta}{\eta(\eta-\zeta)} & =-\frac{1}{\zeta} \\
\frac{1}{2 \pi i} \int_{\gamma} \frac{\omega(\eta)}{\overline{\omega^{\prime}(\eta)}} \cdot \frac{\mathrm{d} \eta}{\eta-\zeta} & =-g_{1}(\zeta)-g_{2}(\zeta)-g_{3}(\zeta) \\
& =-\frac{m}{\zeta}-\frac{M_{0}}{2}\left[\frac{1}{\zeta+i \sqrt{m}}+\frac{1}{\zeta-i \sqrt{m}}\right]
\end{aligned}
$$

and

$$
\begin{aligned}
& \frac{1}{2 \pi i} \int_{\gamma} \frac{\overline{\omega(\eta)}}{\overline{\omega^{\prime}(\eta)}} \cdot \frac{\mathrm{d} \eta}{\eta-\zeta}=-\frac{\overline{\omega(\zeta)}}{\omega^{\prime}(\zeta)}+h_{1}(\zeta)+h_{2}(\zeta)+h_{3}(\zeta) \\
& =-\frac{\overline{\omega(\zeta)}}{\omega^{\prime}(\zeta)}+m \zeta+\frac{M_{0}}{2 m}\left[\frac{1}{\zeta+\frac{i}{\sqrt{m}}}+\frac{1}{\zeta-\frac{i}{\sqrt{m}}}\right]
\end{aligned}
$$


where $g_{1}(\zeta), g_{2}(\zeta)$ and $g_{3}(\zeta)$ are respectively the principal parts of $\left[\omega(\zeta) / \overline{\omega^{\prime}(\zeta)}\right]$ for $\zeta=0$ and $\zeta= \pm i \sqrt{m} ;$ and $h_{1}(\zeta), h_{2}(\zeta)$ and $h_{3}(\zeta)$ are respectively the principal parts of $\left[\overline{\omega(\zeta)} / \omega^{\prime}(\zeta)\right]$ for $\zeta=\infty$ and $\zeta= \pm i / \sqrt{m}$. Substituting equations (25), (26) and (40) (46) into (33) and (34), we have

$$
\begin{aligned}
\phi_{0}(\zeta)= & \frac{R}{2}\left(\sigma_{1}-\sigma_{2}\right) \frac{1}{\zeta} \mathrm{e}^{2 i x}-\frac{R}{4}\left(\sigma_{1}+\sigma_{2}\right) \frac{m}{\zeta} \\
& -\frac{M_{0}}{2}\left[\frac{\frac{R}{4}\left(\sigma_{1}+\sigma_{2}\right)+\overline{\phi_{0}^{\prime}}\left(\frac{i}{\sqrt{m}}\right)}{\zeta+i \sqrt{m}}+\frac{\frac{R}{4}\left(\sigma_{1}+\sigma_{2}\right)+\overline{\phi_{0}^{\prime}}\left(\frac{-i}{\sqrt{m}}\right)}{\zeta-i \sqrt{m}}\right]
\end{aligned}
$$

and

$$
\begin{aligned}
\psi_{0}(\zeta)= & -\frac{R}{4}\left(\sigma_{1}+\sigma_{2}\right)\left[\frac{1}{\zeta}-m \zeta+\frac{\overline{\omega(\zeta)}}{\omega^{\prime}(\zeta)}\right]-\frac{\overline{\omega(\zeta)}}{\omega^{\prime}(\zeta)} \phi_{0}^{\prime}(\zeta) \\
& +\frac{M_{0}}{2 m}\left[\frac{\frac{R}{4}\left(\sigma_{1}+\sigma_{2}\right)+\phi_{0}^{\prime}\left(\frac{-i}{\sqrt{m}}\right)}{\zeta+\frac{i}{\sqrt{m}}}+\frac{\frac{R}{4}\left(\sigma_{1}+\sigma_{2}\right)+\phi_{0}^{\prime}\left(\frac{i}{\sqrt{m}}\right)}{\zeta-\frac{i}{\sqrt{m}}}\right]
\end{aligned}
$$

The differential of equation (47) is readily obtained

$$
\begin{aligned}
\phi_{0}^{\prime}(\zeta)= & -\frac{R}{2}\left(\sigma_{1}-\sigma_{2}\right) \frac{1}{\zeta^{2}} \mathrm{e}^{2 i \alpha}+\frac{R}{4}\left(\sigma_{1}+\sigma_{2}\right) \frac{m}{\zeta^{2}} \\
& +\frac{M_{0}}{2}\left[\frac{\frac{R}{4}\left(\sigma_{1}+\sigma_{2}\right)+\overline{\phi_{0}^{\prime}}\left(\frac{i}{\sqrt{m}}\right)}{(\zeta+i \sqrt{m})^{2}}+\frac{\frac{R}{4}\left(\sigma_{1}+\sigma_{2}\right)+\overline{\phi_{0}^{\prime}}\left(\frac{-i}{\sqrt{m}}\right)}{(\zeta-i \sqrt{m})^{2}}\right]
\end{aligned}
$$

from which

$$
\begin{aligned}
\phi_{0}^{\prime}\left(\frac{i}{\sqrt{m}}\right)= & \phi_{0}^{\prime}\left(\frac{-i}{\sqrt{m}}\right)=\frac{R}{4\left(1-M_{1}^{2}\right)}\left\{\left(\sigma_{1}+\sigma_{2}\right)\left(1-m^{2}\right)+2\left(\sigma_{1}-\sigma_{2}\right) m \mathrm{e}^{2 i x}\right. \\
& \left.-M_{1}\left[\left(\sigma_{1}+\sigma_{2}\right)\left(1-m^{2}\right)+2\left(\sigma_{1}-\sigma_{2}\right) m \mathrm{e}^{-2 i x}\right]\right\}-\frac{R}{4}\left(\sigma_{1}+\sigma_{2}\right)
\end{aligned}
$$

where

$$
M_{1}=\frac{m}{1-m+m^{2}}
$$

Taking into account equation (50) and its conjugate then by substituting equations (47) and (48) into equations (11) and (12), we finally obtain the stress functions

$$
\phi(\zeta)=\frac{R}{4}\left(\sigma_{1}+\sigma_{2}\right)\left(\zeta-\frac{m}{\zeta}\right)+\frac{R}{2}\left(\sigma_{1}-\sigma_{2}\right) \frac{1}{\zeta} \mathrm{e}^{2 i \alpha}-M_{0}\left[\frac{R}{4}\left(\sigma_{1}+\sigma_{2}\right)+\overline{\phi_{0}^{\prime}}\left(\frac{i}{\sqrt{m}}\right)\right] \frac{\zeta}{\zeta^{2}+m}
$$




$$
\begin{aligned}
\psi(\zeta)= & \frac{R}{4}\left(\sigma_{1}+\sigma_{2}\right)\left[m \zeta-\frac{1}{\zeta}-\frac{\overline{\omega(\zeta)}}{\omega^{\prime}(\zeta)}\right]-\frac{R}{2}\left(\sigma_{1}-\sigma_{2}\right) \zeta \mathrm{e}^{2 i x} \\
& -\frac{\overline{\omega(\zeta)}}{\omega^{\prime}(\zeta)} \phi_{0}^{\prime}(\zeta)+M_{0}\left[\frac{R}{4}\left(\sigma_{1}+\sigma_{2}\right)+\phi_{0}^{\prime}\left(\frac{i}{\sqrt{m}}\right)\right] \frac{\zeta}{m \zeta^{2}+1}
\end{aligned}
$$

Equations (52) and (53) together with (49), (50) and (36) are the complete solution of Case 1.

Case 2: uniform pressure on the notch surface profile

Boundary conditions. When the boundary of a lip-shaped notch is subjected to a uniform pressure [Fig. 1(b)], the corresponding boundary conditions are

$$
\Gamma=\Gamma_{1}=0
$$

and

$$
\left.\begin{array}{l}
X_{n}=-P \cos (n, x) \\
Y_{n}=-P \cos (n, y)
\end{array}\right\}
$$

where $P$ is the magnitude of the pressure and $n$ is the exterior normal to the notch profile. Hence the complex boundary conditions are

$$
f=i \int_{\nu}\left(X_{n}+i Y_{n}\right) \mathrm{d} s=-P z=-P R\left[\eta+\frac{m}{\eta}+\frac{\eta}{r^{2}\left(\eta^{2}+m\right)}\right]
$$

and

$$
\bar{f}=-P \bar{z}=-P R\left[m \eta+\frac{1}{\eta}+\frac{\eta}{r^{2}\left(1+m \eta^{2}\right)}\right] .
$$

Complex stress functions. One may assume that, for this kind of first fundamental boundary problem, the stresses and the rotation vanish at infinity. Then, $\phi(\zeta)$ and $\psi(\zeta)$ are holomorphic outside $\gamma$, and

$$
\phi(\infty)=\psi(\infty)=0
$$

By employing the Cauchy integral theorem, we have (for $|\zeta|>1$ )

$$
\begin{aligned}
& \frac{1}{2 \pi i} \int_{\gamma} \frac{\phi(\eta)}{\eta-\zeta} \mathrm{d} \eta=-\phi(\zeta) \\
& \frac{1}{2 \pi i} \int_{\gamma} \frac{\overline{\phi(\eta)}}{\eta-\zeta} \mathrm{d} \eta=0 \\
& \frac{1}{2 \pi i} \int_{\gamma} \frac{\psi(\eta)}{\eta-\zeta} \mathrm{d} \eta=-\psi(\zeta) \\
& \frac{1}{2 \pi i} \int_{\gamma} \frac{\overline{\psi(\eta)}}{\eta-\zeta} \mathrm{d} \eta=0 .
\end{aligned}
$$


Then, from equation (16) and its conjugate, we obtain

$$
\begin{aligned}
& \phi(\zeta)=-\frac{1}{2 \pi i} \int_{\gamma} \frac{f \mathrm{~d} \eta}{\eta-\zeta}+\frac{1}{2 \pi i} \int_{\gamma} \frac{\omega(\eta)}{\overline{\omega^{\prime}(\eta)}} \overline{\phi_{0}^{\prime}(\eta)} \frac{\mathrm{d} \eta}{\eta-\zeta} \\
& \psi(\zeta)=-\frac{1}{2 \pi i} \int_{\gamma} \frac{\bar{f} \mathrm{~d} \eta}{\eta-\zeta}+\frac{1}{2 \pi i} \int_{\gamma} \frac{\overline{\omega(\eta)}}{\omega^{\prime}(\eta)} \phi^{\prime}(\eta) \frac{\mathrm{d} \eta}{\eta-\zeta}
\end{aligned}
$$

As in Case $1\left[\omega(\zeta) / \overline{\omega^{\prime}(\zeta)}\right] \overline{\phi^{\prime}}(1 / \zeta)$ is judged to be holomorphic inside $\gamma$, except at $\zeta= \pm i \sqrt{m}$; and $\left[\overline{\omega(\zeta)} / \omega^{\prime}(\zeta)\right] \phi^{\prime}(\zeta)$ is holomorphic outside $\gamma$, except at $\zeta= \pm i / \sqrt{m}$. According to the Cauchy integral theorem, one finds (for $|\zeta|>1$ )

$$
\begin{aligned}
\frac{1}{2 \pi i} \int_{\gamma} \frac{\omega(\eta)}{\overline{\omega^{\prime}(\eta)}} \overline{\phi^{\prime}(\eta)} \frac{\mathrm{d} \eta}{\eta-\zeta} & =\frac{1}{2 \pi i} \int_{\gamma} \frac{\omega(\eta)}{\overline{\omega^{\prime}(\eta)}} \overline{\phi^{\prime}}\left(\frac{1}{\eta}\right) \frac{\mathrm{d} \eta}{\eta-\zeta} \\
& =-G_{1}(\zeta)-G_{2}(\zeta)=-\frac{M_{0}}{2}\left[\frac{\overline{\phi^{\prime}}\left(\frac{-i}{\sqrt{m}}\right)}{\zeta-i \sqrt{m}}+\frac{\overline{\phi^{\prime}}\left(\frac{i}{\sqrt{m}}\right)}{\zeta+i \sqrt{m}}\right]
\end{aligned}
$$

where $G_{1}(\zeta)$ and $G_{2}(\zeta)$ are respectively the principal parts of $\left[\omega(\zeta) \sqrt{\omega^{\prime}(\zeta)}\right] \overline{\phi^{\prime}(\zeta)}$ for $\zeta= \pm i \sqrt{m}$; and

$$
\begin{aligned}
\frac{1}{2 \pi i} \int_{\gamma} \frac{\overline{\omega(\eta)}}{\omega^{\prime}(\eta)} \phi^{\prime}(\eta) \frac{\mathrm{d} \eta}{\eta-\zeta} & =-\frac{\overline{\omega(\zeta)}}{\omega^{\prime}(\zeta)} \phi^{\prime}(\zeta)+H_{1}(\zeta)+H_{2}(\zeta) \\
& =-\frac{\overline{\omega(\zeta)}}{\omega^{\prime}(\zeta)} \phi^{\prime}(\zeta)+\frac{M_{0}}{2 m}\left[\frac{\phi^{\prime}\left(\frac{i}{\sqrt{m}}\right)}{\zeta-\frac{i}{\sqrt{m}}}+\frac{\phi^{\prime}\left(\frac{-i}{\sqrt{m}}\right)}{\zeta+\frac{i}{\sqrt{m}}}\right]
\end{aligned}
$$

where $H_{1}(\zeta)$ and $H_{2}(\zeta)$ are respectively the principal parts of $\left[\overline{\omega(\zeta)} / \omega^{\prime}(\zeta)\right] \phi^{\prime}(\zeta)$ for $\zeta= \pm i / \sqrt{m}$. Note that $\left[\overline{\omega(\zeta)} / \omega^{\prime}(\zeta)\right]$ and $M_{0}$ are given in equations (36) and (42).

In order to solve the first term on the right hand side of equations (62) and (63), we again use the Cauchy integral theorem (for $|\zeta|>1$ )

$$
\frac{1}{2 \pi i} \int_{\gamma} \frac{\eta}{\eta^{2}+m} \cdot \frac{\mathrm{d} \eta}{\eta-\zeta}=-\frac{\zeta}{\zeta^{2}+m}
$$

and

$$
\frac{1}{2 \pi i} \int_{\gamma} \frac{\eta}{1+m \eta^{2}} \cdot \frac{\mathrm{d} \eta}{\eta-\zeta}=0 .
$$

Substituting equations (56) and (57) into (62) and (63), and using the above integral formulae as well as those of equations (43) and (44), we obtain

$$
\phi(\zeta)=-P R\left[\frac{m}{\zeta}+\frac{\zeta}{r^{2}\left(\zeta^{2}+m\right)}\right]-\frac{M_{0}}{2}\left[\frac{\overline{\phi^{\prime}}\left(\frac{-i}{\sqrt{m}}\right)}{\zeta-i \sqrt{m}}+\frac{\bar{\phi}^{\prime}\left(\frac{i}{\sqrt{m}}\right)}{\zeta+i \sqrt{m}}\right]
$$


and

$$
\psi(\zeta)=-P R \frac{1}{\zeta}-\frac{\overline{\omega(\zeta)}}{\omega^{\prime}(\zeta)} \phi^{\prime}(\zeta)+\frac{M_{0}}{2 m}\left[\frac{\phi^{\prime}\left(\frac{i}{\sqrt{m}}\right)}{\zeta-\frac{i}{\sqrt{m}}}+\frac{\phi^{\prime}\left(\frac{-i}{\sqrt{m}}\right)}{\zeta+\frac{i}{\sqrt{m}}}\right]
$$

From the differential of equation (68), we obtain

$$
\phi^{\prime}\left(\frac{i}{\sqrt{m}}\right)=\phi^{\prime}\left(\frac{-i}{\sqrt{m}}\right)=\overline{\phi^{\prime}}\left(\frac{i}{\sqrt{m}}\right)=\overline{\phi^{\prime}}\left(\frac{-i}{\sqrt{m}}\right)=-P R M_{3}
$$

where

$$
M_{3}=\frac{m\left(1-m+m^{2}\right)\left(1+m-m^{2}+m^{3}\right)}{\left(1+m^{2}\right)(1-m)^{2}} .
$$

Therefore the complex stress functions are

$$
\begin{aligned}
& \phi(\zeta)=-P R\left\{\frac{m}{\zeta}+\frac{\zeta}{\zeta^{2}+m}(1+m)^{2}\left[1-\frac{m\left(1+m-m^{2}+m^{3}\right)}{\left(1+m^{2}\right)^{2}}\right]\right\} \\
& \psi(\zeta)=-P R\left\{\frac{1}{\zeta}+\frac{\zeta}{1+m \zeta^{2}}\left[\frac{m(1+m)^{2}\left(1+m-m^{2}+m^{3}\right)}{\left(1+m^{2}\right)^{2}}\right]\right\}-\frac{\overline{\omega(\zeta)}}{\omega^{\prime}(\zeta)} \phi^{\prime}(\zeta)
\end{aligned}
$$

and hence this problem is solved.

\section{Case 3: uniform pressure on part of a notch boundary}

Boundary conditions. When a part of a notch boundary that is symmetric to the $y$-axis, is subjected to a uniform pressure (Fig. 4), the corresponding boundary conditions are

$$
\left.\begin{array}{c}
\Gamma=\Gamma_{1}=0 \\
f=-P z=-P R\left[\eta+\frac{m}{\eta}+\frac{\eta}{r^{2}\left(\eta^{2}+m\right)}\right] \\
f=-P z_{2} \\
\text { for } z_{1} \text { to } i \text { to } z_{2} \\
\text { for } z_{2} \text { to }-i \text { to } z_{1}
\end{array}\right\}
$$

and

$$
X+i Y=i P\left(z_{1}-z_{2}\right)
$$

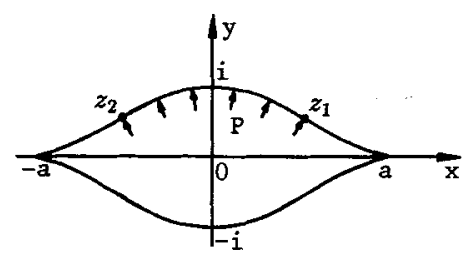

(a) $z$-plane



(b) $\zeta$-plane

Fig. 4. Uniform pressure on part of a notch boundary profile. 
Since $z_{1}$ and $z_{2}$ are symmetric with respect to the $y$-axis, it follows that

$$
X+i Y=2 i P x_{1}
$$

and

$$
X-i Y=-2 i P x_{1} .
$$

Therefore equations (20) and (21) reduce to

$$
f_{0}=f+\frac{i}{\pi} P x_{1} \ln \eta-\frac{i P x_{1}}{\pi(1+\kappa)} \cdot \frac{\omega(\eta)}{\omega^{\prime}(\eta)} \cdot \eta
$$

and

$$
\overline{f_{0}}=\bar{f}+\frac{i}{\pi} P x_{1} \ln \eta+\frac{i P x_{1}}{\pi(1+\kappa)} \cdot \frac{\overline{\omega(\eta)}}{\omega^{\prime}(\eta)} \cdot \frac{1}{\eta}
$$

Complex stress functions. Substituting equations (78) and (79) into (33) and (34), we have

$$
\begin{aligned}
\phi_{0}(\zeta)= & -\frac{1}{2 \pi i} \int_{\gamma} \frac{f \mathrm{~d} \eta}{\eta-\zeta}-\frac{i}{\pi} P x_{1} \frac{1}{2 \pi i} \int_{\gamma} \frac{\ln \eta}{\eta-\zeta} \mathrm{d} \eta \\
& +\frac{i P x_{1}}{\pi(1+\kappa)} \cdot \frac{1}{2 \pi i} \int_{\nu} \frac{\omega(\eta)}{\overline{\omega^{\prime}(\eta)}} \cdot \frac{\eta \mathrm{d} \eta}{\eta-\zeta}+\frac{1}{2 \pi i} \int_{\nu} \frac{\omega(\eta)}{\overline{\omega^{\prime}(\eta)}} \overline{\phi_{0}^{\prime}(\eta)} \frac{\mathrm{d} \eta}{\eta-\zeta}
\end{aligned}
$$

and

$$
\begin{aligned}
\psi_{0}(\zeta)= & -\frac{1}{2 \pi i} \int_{\gamma} \frac{\bar{f} \mathrm{~d} \eta}{\eta-\zeta}-\frac{i}{\pi} P x_{1} \frac{1}{2 \pi i} \int_{\gamma} \frac{\ln \eta}{\eta-\zeta} \mathrm{d} \eta \\
& -\frac{i P x_{1}}{\pi(1+\kappa)} \cdot \frac{1}{2 \pi i} \int_{\gamma} \frac{\overline{\omega(\eta)}}{\omega^{\prime}(\eta)} \cdot \frac{\mathrm{d} \eta}{\eta(\eta-\zeta)}+\frac{1}{2 \pi i} \int_{\gamma} \frac{\overline{\omega(\eta)}}{\omega^{\prime}(\eta)} \phi_{0}^{\prime}(\eta) \frac{\mathrm{d} \eta}{\eta-\zeta} .
\end{aligned}
$$

Using the boundary condition of equation (75), we write the first integral of the above equations as

$$
-\frac{1}{2 \pi i} \int_{\gamma} \frac{f \mathrm{~d} \eta}{\eta-\zeta}=\frac{P R}{2 \pi i} \int_{\eta_{1}}^{\eta_{2}}\left[\eta+\frac{m}{\eta}+\frac{\eta}{r^{2}\left(\eta^{2}+m\right)}\right] \frac{\mathrm{d} \eta}{\eta-\zeta}+\frac{P z_{2}}{2 \pi i} \int_{\eta_{2}}^{\eta_{1}} \frac{\mathrm{d} \eta}{\eta-\zeta}
$$

and

$$
-\frac{1}{2 \pi i} \int_{\gamma} \frac{\bar{f} \mathrm{~d} \eta}{\eta-\zeta}=\frac{P R}{2 \pi i} \int_{\eta_{1}}^{\eta_{2}}\left[m \eta+\frac{1}{\eta}+\frac{\eta}{r^{2}\left(1+m \eta^{2}\right)}\right] \frac{\mathrm{d} \eta}{\eta-\zeta}+\frac{P \overline{z_{2}}}{2 \pi i} \int_{\eta_{2}}^{\eta_{1}} \frac{\mathrm{d} \eta}{\eta-\zeta} .
$$

This is followed by showing that

$$
\int_{\eta_{1}}^{\eta_{2}}\left(\eta+\frac{m}{\eta}\right) \frac{\mathrm{d} \eta}{\eta-\zeta}=\eta_{2}-\eta_{1}+\left(\zeta+\frac{m}{\zeta}\right) \ln \frac{\eta_{2}-\zeta}{\eta_{1}-\zeta}-\frac{m}{\zeta} \ln \frac{\eta_{2}}{\eta_{1}}
$$

and

$$
\begin{aligned}
\int_{\eta_{1}}^{\eta_{2}} \frac{\eta}{\eta^{2}+m} \cdot \frac{\mathrm{d} \eta}{\eta-\zeta}= & \frac{b_{0}}{\sqrt{m}}\left(\arctan \frac{\eta_{2}}{\sqrt{m}}-\arctan \frac{\eta_{1}}{\sqrt{m}}\right) \\
& -\frac{c_{0}}{2} \ln \frac{\eta_{2}^{2}+m}{\eta_{1}^{2}+m}+c_{0} \ln \frac{\eta_{2}-\zeta}{\eta_{1}-\zeta}
\end{aligned}
$$


where

$$
b_{0}=\frac{m}{\zeta^{2}+m} \text { and } \quad c_{0}=\frac{\zeta}{\zeta^{2}+m}
$$

whereas

$$
\int_{\eta_{1}}^{\eta_{2}}\left(m \eta+\frac{1}{\eta}\right) \frac{\mathrm{d} \eta}{\eta-\zeta}=m\left(\eta_{2}-\eta_{1}\right)+\left(m \zeta+\frac{1}{\zeta}\right) \ln \frac{\eta_{2}-\zeta}{\eta_{1}-\zeta}-\frac{1}{\zeta} \ln \frac{\eta_{2}}{\eta_{1}}
$$

and

$$
\begin{aligned}
\int_{\eta_{1}}^{\eta_{2}} \frac{\eta}{1+m \eta^{2}} \cdot \frac{\mathrm{d} \eta}{\eta-\zeta}= & \frac{b_{1}}{\sqrt{m}}\left[\arctan \left(\eta_{2} \sqrt{m}\right)-\arctan \left(\eta_{1} \sqrt{m}\right)\right] \\
& -\frac{c_{1}}{2} \ln \frac{1+m \eta_{2}^{2}}{1+m \eta_{1}^{2}}+c_{1} \ln \frac{\eta_{2}-\zeta}{\eta_{1}-\zeta}
\end{aligned}
$$

where

$$
b_{1}=\frac{1}{1+m \zeta^{2}} \quad \text { and } \quad c_{1}=\frac{\zeta}{1+m \zeta^{2}} .
$$

Subsequently, we solve the following integrals for the second and third terms on the right hand side of equations (80) and (81)

$$
\begin{aligned}
& \frac{1}{2 \pi i} \int_{\gamma} \frac{\ln \eta}{\eta-\zeta} \mathrm{d} \eta=\ln \left(\eta_{1}-\zeta\right)-\ln \zeta \\
& \frac{1}{2 \pi i} \int_{\nu} \frac{\omega(\eta)}{\frac{\omega^{\prime}(\eta)}{\eta}} \cdot \frac{\eta \mathrm{d} \eta}{\eta-\zeta}=\frac{m\left(1-m^{2}\right)^{2}}{\left(1+m^{2}\right)\left(1-m+m^{2}\right)} \cdot \frac{1}{\zeta^{2}+m}
\end{aligned}
$$

and

$$
\frac{1}{2 \pi i} \int_{\nu} \frac{\overline{\omega(\eta)}}{\overline{\omega^{\prime}(\eta)}} \cdot \frac{\mathrm{d} \eta}{\eta(\eta-\zeta)}=-\frac{\overline{\omega(\zeta)}}{\omega^{\prime}(\zeta)} \cdot \frac{1}{\zeta}+\frac{\left(1-m^{2}\right)^{2}}{\left(1+m^{2}\right)\left(1-m+m^{2}\right)} \cdot \frac{1}{1+m \zeta^{2}}
$$

The fourth term on the right hand side of equations (80) and (81) respectively takes the same form as equations (40) and (41). Thus, we have

$$
\phi_{0}(\zeta)=\frac{P}{2 \pi} I_{0}(\zeta)-\frac{M_{0}}{2}\left[\frac{\overline{\phi_{0}^{\prime}}\left(\frac{-i}{\sqrt{m}}\right)}{\zeta-i \sqrt{m}}+\frac{\overline{\phi_{0}^{\prime}}\left(\frac{i}{\sqrt{m}}\right)}{\zeta+i \sqrt{m}}\right]
$$

where

$$
\begin{aligned}
I_{0}(\zeta)= & \frac{R}{i}\left\{\eta_{2}-\eta_{1}+\left(\zeta+\frac{m}{\zeta}\right) \ln \frac{\eta_{2}-\zeta}{\eta_{1}-\zeta}-\frac{m}{\zeta} \ln \frac{\eta_{2}}{\eta_{1}}\right. \\
& \left.+(1+m)^{2}\left[\frac{b_{0}}{\sqrt{m}}\left(\arctan \frac{\eta_{2}}{\sqrt{m}}-\arctan \frac{\eta_{1}}{\sqrt{m}}\right)-\frac{c_{0}}{2} \ln \frac{\eta_{2}^{2}+m}{\eta_{1}^{2}+m}+c_{0} \ln \frac{\eta_{2}-\zeta}{\eta_{1}-\zeta}\right]\right\} \\
& +\frac{z_{2}}{i} \ln \frac{\eta_{1}-\zeta}{\eta_{2}-\zeta}-2 i x_{1} \ln \frac{\eta_{1}-\zeta}{\zeta}+\frac{2 i x_{1} M_{0}}{1+\kappa} \cdot \frac{m}{\zeta^{2}+m}
\end{aligned}
$$


and

$$
\psi_{0}(\zeta)=\frac{P}{2 \pi} I_{1}(\zeta)-\frac{\overline{\omega(\zeta)}}{\omega^{\prime}(\zeta)} \phi_{0}^{\prime}(\zeta)+\frac{M_{0}}{2 m}\left[\frac{\phi_{0}^{\prime}\left(\frac{i}{\sqrt{m}}\right)}{\zeta-\frac{i}{\sqrt{m}}}+\frac{\phi_{0}^{\prime}\left(\frac{-i}{\sqrt{m}}\right)}{\zeta+\frac{i}{\sqrt{m}}}\right]
$$

where

$$
\begin{aligned}
I_{1}(\zeta)= & \frac{R}{i}\left\{m\left(\eta_{2}-\eta_{1}\right)+\left(m \zeta+\frac{1}{\zeta}\right) \ln \frac{\eta_{2}-\zeta}{\eta_{1}-\zeta}-\frac{1}{\zeta} \ln \frac{\eta_{2}}{\eta_{1}}\right. \\
& \left.+(1+m)^{2}\left[\frac{b_{1}}{\sqrt{m}}\left[\arctan \left(\eta_{2} \sqrt{m}\right)-\arctan \left(\eta_{1} \sqrt{m}\right)\right]-\frac{c_{1}}{2} \ln \frac{1+m \eta_{2}^{2}}{1+m \eta_{1}^{2}}+c_{1} \ln \frac{\eta_{2}-\zeta}{\eta_{1}-\zeta}\right]\right\} \\
& +\frac{\overline{z_{2}}}{i} \ln \frac{\eta_{1}-\zeta}{\eta_{2}-\zeta}-2 i x_{1} \ln \frac{\eta_{1}-\zeta}{\zeta}+\frac{2 i x_{1}}{1+\kappa}\left[\frac{\overline{\omega(\zeta)}}{\omega^{\prime}(\zeta)} \cdot \frac{1}{\zeta}-\frac{M_{0}}{1+m \eta^{2}}\right]
\end{aligned}
$$

and $M_{0}$ is provided by equation (42). The differential of equation (91) gives

$$
\phi_{0}^{\prime}(\zeta)=\frac{P}{2 \pi} I_{0}^{\prime}(\zeta)+\frac{M_{0}}{2}\left[\frac{\overline{\phi_{0}^{\prime}}\left(\frac{-i}{\sqrt{m}}\right)}{(\zeta-i \sqrt{m})^{2}}+\frac{\overline{\phi_{0}^{\prime}}\left(\frac{i}{\sqrt{m}}\right)}{(\zeta+i \sqrt{m})^{2}}\right]
$$

where

$$
\begin{aligned}
I_{0}^{\prime}(\zeta)= & -i R\left\{\left(1-\frac{m}{\zeta^{2}}\right) \ln \frac{\eta_{2}-\zeta}{\eta_{1}-\zeta}+\left(\zeta+\frac{m}{\zeta}\right) \frac{\eta_{2}-\eta_{1}}{\left(\eta_{2}-\zeta\right)\left(\eta_{1}-\zeta\right)}+\frac{m}{\zeta^{2}} \ln \frac{\eta_{2}}{\eta_{1}}\right. \\
& +(1+m)^{2}\left[\frac{2 \zeta \sqrt{m}}{\left(\zeta^{2}+m\right)^{2}}\left(\arctan \frac{\eta_{1}}{\sqrt{m}}-\arctan \frac{\eta_{2}}{\sqrt{m}}\right)\right. \\
& \left.\left.+\frac{\zeta^{2}-m}{\left(\zeta^{2}+m\right)^{2}}\left(\frac{1}{2} \ln \frac{\eta_{2}^{2}+m}{\eta_{1}^{2}+m}-\ln \frac{\eta_{2}-\zeta}{\eta_{1}-\zeta}\right)+\frac{\zeta}{\zeta^{2}+m} \cdot \frac{\eta_{2}-\eta_{1}}{\left(\eta_{2}-\zeta\right)\left(\eta_{1}-\zeta\right)}\right]\right\} \\
& +i z_{2} \frac{\eta_{2}-\eta_{1}}{\left(\eta_{2}-\zeta\right)\left(\eta_{1}-\zeta\right)}+2 i x_{1}\left[\frac{\eta_{1}}{\zeta\left(\eta_{1}-\zeta\right)}-\frac{2 M_{0}}{1+\kappa} \cdot \frac{m \zeta}{\left(\zeta^{2}+m\right)^{2}}\right]
\end{aligned}
$$

from equation (95), the constant coefficients are deduced as follows

$$
\left.\begin{array}{l}
\phi_{0}^{\prime}\left(\frac{i}{\sqrt{m}}\right)=\phi_{0}^{\prime}\left(\frac{-i}{\sqrt{m}}\right)=\frac{P}{2 \pi\left(1-M_{1}^{2}\right)}\left[I_{0}^{\prime}\left(\frac{i}{\sqrt{m}}\right)-M_{1} \overline{I_{0}^{\prime}}\left(\frac{i}{\sqrt{m}}\right)\right] \\
\overline{\phi_{0}^{\prime}}\left(-\frac{i}{\sqrt{m}}\right)=\overline{\phi_{0}^{\prime}}\left(\frac{-i}{\sqrt{m}}\right)=\frac{P}{2 \pi\left(1-M_{1}^{2}\right)}\left[\overline{I_{0}^{\prime}}\left(\frac{i}{\sqrt{m}}\right)-M_{1} I_{0}^{\prime}\left(\frac{i}{\sqrt{m}}\right)\right]
\end{array}\right\}
$$


where $M_{1}$ is given by equation (51). Inserting equations (76), (77), (91), (93) and (97) into (11) and (12), we therefore obtain the complex stress functions for this problem

$$
\begin{aligned}
& \phi(\zeta)=-\frac{i P x_{1}}{\pi(1+\kappa)} \ln \zeta+\frac{P}{2 \pi} I_{0}(\zeta)-M_{0} \frac{\zeta}{\zeta^{2}+M} \overline{\phi_{0}^{\prime}}\left(\frac{i}{\sqrt{m}}\right) \\
& \psi(\zeta)=-\frac{i \kappa P x_{1}}{\pi(1+\kappa)} \ln \zeta+\frac{P}{2 \pi} I_{1}(\zeta)-\frac{\overline{\omega(\zeta)}}{\omega^{\prime}(\zeta)} \phi_{0}^{\prime}(\zeta)+M_{0} \frac{\zeta}{m^{2}+1} \phi_{0}^{\prime}\left(\frac{i}{\sqrt{m}}\right)
\end{aligned}
$$

\section{APPLICATIONS}

With the previously derived complex stress functions, one may calculate, by using the following formulae, the stress distributions of plates containing central lip-shaped notches under different loading conditions

$$
\left.\begin{array}{c}
\sigma_{x}+\sigma_{y}=4 \operatorname{Re}[\Phi(\zeta)] \\
\sigma_{y}-\sigma_{x}+2 i \tau_{x y}=\frac{2}{\omega^{\prime}(\zeta)}\left[\Phi^{\prime}(\zeta) \overline{\omega(\zeta)}+\Psi(\zeta) \omega^{\prime}(\zeta)\right]
\end{array}\right\}
$$

where

$$
\Phi(\zeta)=\frac{\phi^{\prime}(\zeta)}{\omega^{\prime}(\zeta)} \text { and } \Psi(\zeta)=\frac{\psi^{\prime}(\zeta)}{\omega^{\prime}(\zeta)}
$$

However, here we discuss the stress intensity factors at cracked lip-shaped notches, which are themselves deduced from the complex stress functions, together with their applications to other notch-crack configurations.

\section{Stress intensity factors for lip-shaped notches}

The relationship between stress intensity factors $\left(K_{\mathrm{I}}\right.$ and $\left.K_{\mathrm{II}}\right)$ and a complex stress function takes the form [10]

$$
K_{\mathrm{I}}-i K_{\mathrm{II}}=2 \sqrt{\pi} \phi^{\prime}(1)\left[\omega^{\prime \prime}(1)\right]^{-1 / 2} .
$$

Referring to equations (4), (5) and (3), we write

$$
\omega^{\prime \prime}(1)=2 R \frac{(1-m)^{2}}{1+m}=a\left(\frac{1-m}{1+m}\right)^{2} \text {. }
$$

Remote biaxial loading. For the case of a central lip-shape notched plate under remote biaxial loading, we use equation (52) and the conjugate of (50) to obtain

$$
\phi^{\prime}(1)=\frac{R}{4}\left(\sigma_{1}+\sigma_{2}\right)(1+m)-\frac{R}{2}\left(\sigma_{1}-\sigma_{2}\right) \mathrm{e}^{2 i \alpha}+M_{2}\left[\frac{R}{4}\left(\sigma_{1}+\sigma_{2}\right)+\overline{\phi_{0}^{\prime}}\left(\frac{i}{\sqrt{m}}\right)\right]
$$

where

$$
M_{2}=\frac{(1-m)^{3}}{\left(1+m^{2}\right)\left(1-m+m^{2}\right)}
$$


As an example, we let $\alpha=\pi / 2$ and consider three typical degrees of biaxiality: $\lambda=0$ (uniaxial), 1.0 (equibiaxial) and -1.0 (shear), where $\lambda=\sigma_{2} / \sigma_{1}$. When $\lambda=0$, i.e. $\sigma_{2}=0$, by inserting equation (103) into (101), we find

$$
\begin{gathered}
K_{\mathrm{I}}=\sigma_{1} \sqrt{\pi a}\left(1+F_{0}\right) \\
K_{\mathrm{II}}=0 \\
F_{0}=\frac{m^{2}\left(3+3 m-m^{2}+3 m^{3}\right)}{2(1-m)\left(1+m^{2}\right)^{2}} .
\end{gathered}
$$

When $\lambda=1$, i.e. $\sigma_{2}=\sigma_{1}$, we have

$$
\begin{aligned}
K_{\mathrm{I}} & =\sigma_{1} \sqrt{\pi a}\left(1+F_{1}\right) \\
K_{\mathrm{II}} & =0
\end{aligned}
$$

where

$$
F_{1}=\frac{2 m^{3}\left(2-m+m^{2}\right)}{(1-m)\left(1+m^{2}\right)^{2}} .
$$

When $\lambda=-1$, i.e. $\sigma_{2}=-\sigma_{1}$, we obtain

$$
\begin{aligned}
K_{\mathrm{I}} & =\sigma_{1} \sqrt{\pi a}\left(1+F_{-1}\right) \\
K_{\mathrm{II}} & =0
\end{aligned}
$$

where

$$
F_{-1}=\frac{m^{2}\left(3-m+m^{2}+m^{3}\right)}{(1-m)\left(1+m^{2}\right)^{2}}
$$

Figure 5 shows the results of equations (105), (107) and (109), which describe the variation of $K_{\mathrm{I}} /\left(\sigma_{1} \sqrt{\pi a}\right)$ with $a$ increasing from $b$ to $\infty$, at different biaxialities.

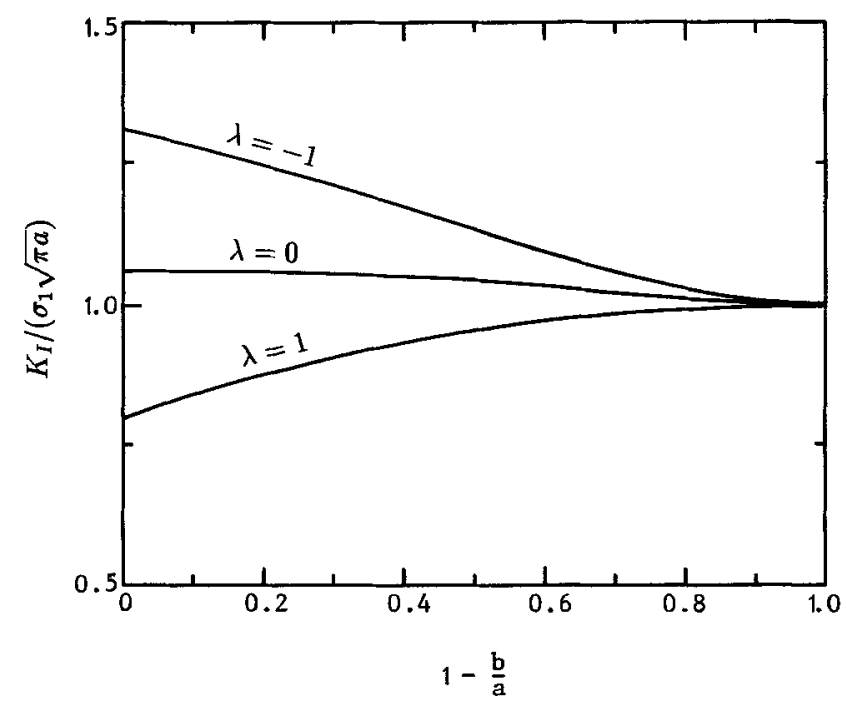

Fig. 5. Variation of $K_{1} /\left(\sigma_{1} \sqrt{\pi a}\right)$ with $(1-b / a)$ for lip-shaped notches in infinite plates at three biaxialities. 
It is obvious that, by referring to equation (7), if $a \gg 1$ (i.e. the notch tends to be a sharp crack), then $m \rightarrow 0^{-}$, and

$$
F_{0} \rightarrow 0^{+}, \quad F_{1} \rightarrow 0^{-} \text {and } f_{-1} \rightarrow 0^{+} .
$$

As a result, equations (105), (107) and (109) all reduce to

$$
K_{\mathrm{I}}=\sigma_{1} \sqrt{\pi a}
$$

which indicates that the extremity of the present results decays to the solution of an infinite plate containing a central through crack.

Uniform pressure on notch boundary. When a plate containing a central lip-shaped notch is subjected to uniform pressure on its notch profile, we have, from equation (72)

$$
\phi^{\prime}(1)=P R\left[1-\frac{m(1-m)\left(1+m-m^{2}+m^{3}\right)}{\left(1+m^{2}\right)^{2}}\right] .
$$

Substituting equations (112) and (102) into (101), we obtain

$$
\begin{aligned}
K_{\mathrm{I}} & =P \sqrt{\pi a}\left(1+F_{\mathrm{p}}\right) \\
K_{\mathrm{II}} & =0
\end{aligned}
$$

where

$$
F_{\mathrm{p}}=\frac{2 m^{3}\left(2-m+m^{2}\right)}{(1-m)\left(1+m^{2}\right)^{2}}
$$

Note that, $F_{\mathrm{p}}=F_{1}$. In other words, the stress intensity factor for a lip-shaped notch in a plate subjected to remote equibiaxial tension, is identical with that of a similar notch with its profile subjected to a uniform pressure of the same magnitude. This implies that Bueckner's principle [12] is also applicable to this notch problem.

\section{Cracks originating from a circular or an elliptical notch}

If we make a replacement for a series of lip-shaped notches of different ratios of $a / b$ by a circle of radius $d=b$, and adding two slits of length $l$ corresponding to $(a-d)$ between $x= \pm d$ and $\pm a$ [e.g. Fig. 3(a)], then we observe that, after $a / d \geqslant 1.4$, the results yielded by lip-shaped notches are consistent with the numerical data produced from a circular notch containing two cracks [Fig. 6(a)]. In addition, if lip-shaped notches are replaced by an ellipse of $b / d=0.5$ with two cracks of corresponding length [e.g. Fig. 3(b)], after $a / d \geqslant 1.2$, the present results also fit the related numerical data [Fig. 6(b)]. It seems that, in general, when

$$
\frac{a-d}{b} \geqslant 0.4
$$

the stress intensity factors of lip-shaped notch cracks are approximately equal to those for a pair of symmetrical cracks emanating from a circular or an elliptical notch. This suggests that, for the concerned configurations, the sensitive factor affecting $K_{\mathrm{I}}$ and $K_{\mathrm{II}}$ is the total length of crack plus notch radius, whereas the notch shape itself is less sensitive. In this sense, the present analytical solutions and the simple $K$-expressions can be used to calculate stress intensity factors for cracks emanating from a circular or an elliptical notch. The above statement is illustrated by Fig. 7, indicating that three configurations of the same total notch-crack length $2 a$ and notch width $2 b$, possess the same stress intensity factor at the notch-crack tip. Summing up equations (105), (107) 



Fig. 6. Comparison of present results to numerical data of matched configurations. (a) Cracks emánating from a circular notch $(b / d=1.0)$. $O: \lambda=0$, average of data from Refs $[1,2,4,6] ; \triangle: \lambda=1$, average of data from $\operatorname{Refs}[1,2,6] ; \nabla:$ $\lambda=-1$, average of data from Refs [2] and [6]. (b) Cracks emanating from an elliptical notch $(b / d=0.5) . \bigcirc: \lambda=0$, average of data from $\operatorname{Refs}[2,4,6] ; \triangle: \lambda=1$, data from

Ref. [6]; $\nabla: \lambda=-1$, data from Ref. [6].

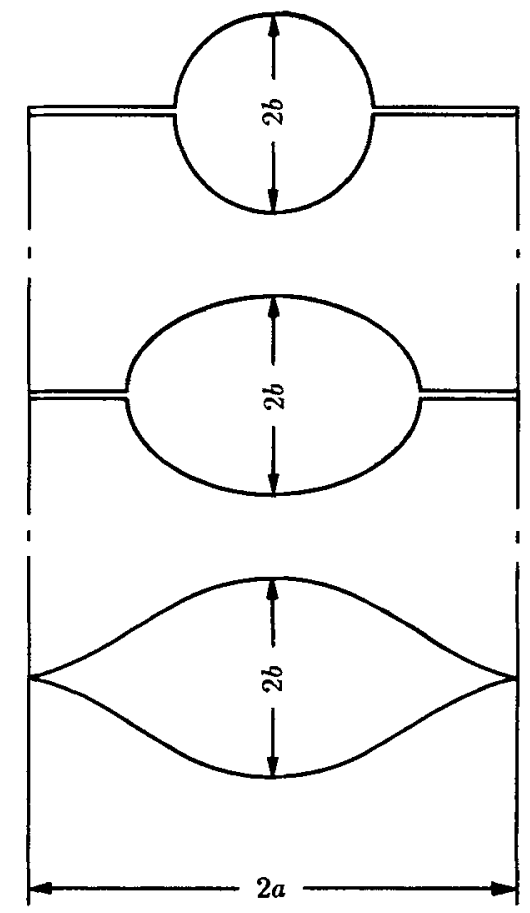

Fig. 7. Three notch-crack configurations possessing approximately the same stress intensity factors.

and (109), we write

where

$$
\begin{aligned}
K_{1} & =\sigma_{1} \sqrt{\pi a}(1+F) \\
K_{\mathrm{II}} & =0
\end{aligned}
$$

$$
F=\frac{m^{2}}{2(1-m)\left(1+m^{2}\right)^{2}}\left[\left(3+3 m-m^{2}+3 m^{3}\right)-\lambda\left(3-5 m+3 m^{2}-m^{3}\right)\right] .
$$

Equation (116) is a useful expression of the stress intensity factor for a notch crack $(\alpha=\pi / 2)$ under various biaxial loading conditions.

Plastic zone sizes

Invoking the Dugdale strip yield concept [11], we assume the following model to evaluate the plastic zone sizes for cracks emanating from a circular notch in an infinite plate subjected to 


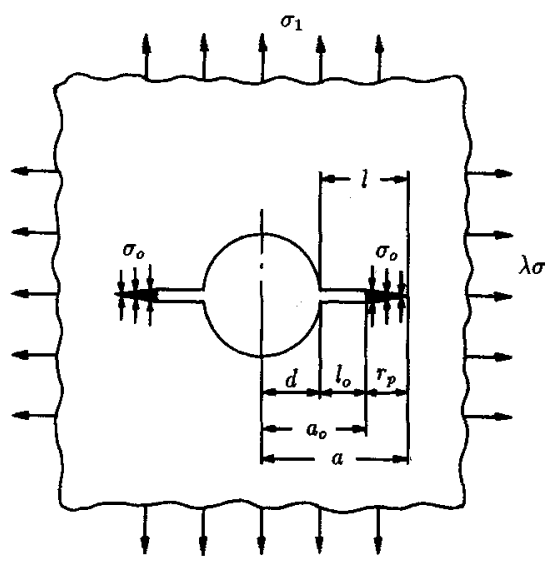

(a) Total problem

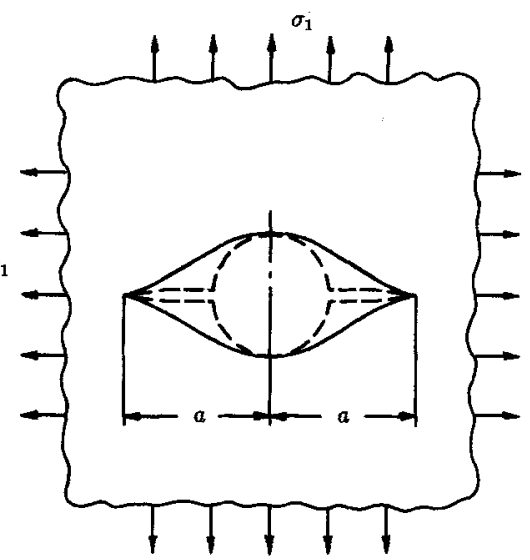

(b) Case A

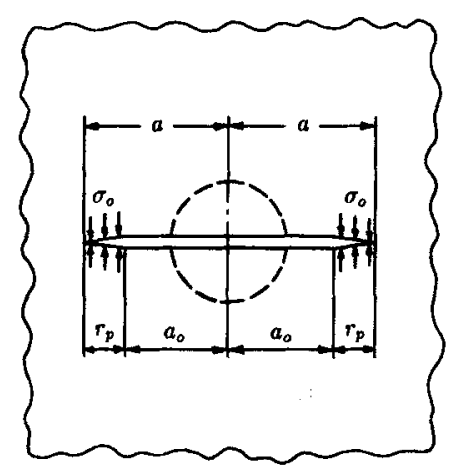

(c) Case B

Fig. 8. Proposed model for plastic zone size determination.

external biaxial loading. As shown in Fig. 8, the problem is created by the linear superposition of Case A (an infinite plate containing a central lip-shaped notch under external biaxial loading [Fig. 8(b)]), and Case B (an infinite plate containing a central through crack, with cohesive stress, $\sigma_{0}$, distributed over two plastic enclave portions [Fig. 8(c)]).

For Case A, referring to previous work, we have

$$
K_{\mathrm{I}}^{\mathrm{A}}=\sigma_{1} \sqrt{\pi a}(1+F)
$$

whereas for Case B, the following well-known solution is introduced

$$
K_{\mathrm{I}}^{\mathrm{B}}=-2 \sigma_{0} \sqrt{\frac{a}{\pi}} \arccos \frac{a_{0}}{a} .
$$

For the total problem, the resulting stress intensity factor at the plastic enclave tip vanishes, i.e.

$$
K_{\mathrm{I}}^{\mathrm{A}}+K_{\mathrm{I}}^{\mathrm{B}}=0 .
$$

Substituting equations (118) and (119) into (120), we have

$$
\frac{\sigma_{1}}{\sigma_{0}}=\frac{2}{\pi} \cdot \frac{1}{1+F} \arccos \frac{a_{0}}{a} .
$$

Regarding the three biaxialities of $\lambda=0,1$ and -1 , for instance, we can write the above equation as

$$
\frac{\sigma_{1}}{\sigma_{0}}=\frac{2}{\pi} \cdot \frac{1}{1+F_{j}} \arccos \frac{a_{0}}{a}
$$

where

$$
F_{j}= \begin{cases}F_{0} & \lambda=0 \\ F_{1} & \lambda=1 \\ F_{-1} & \lambda=-1\end{cases}
$$



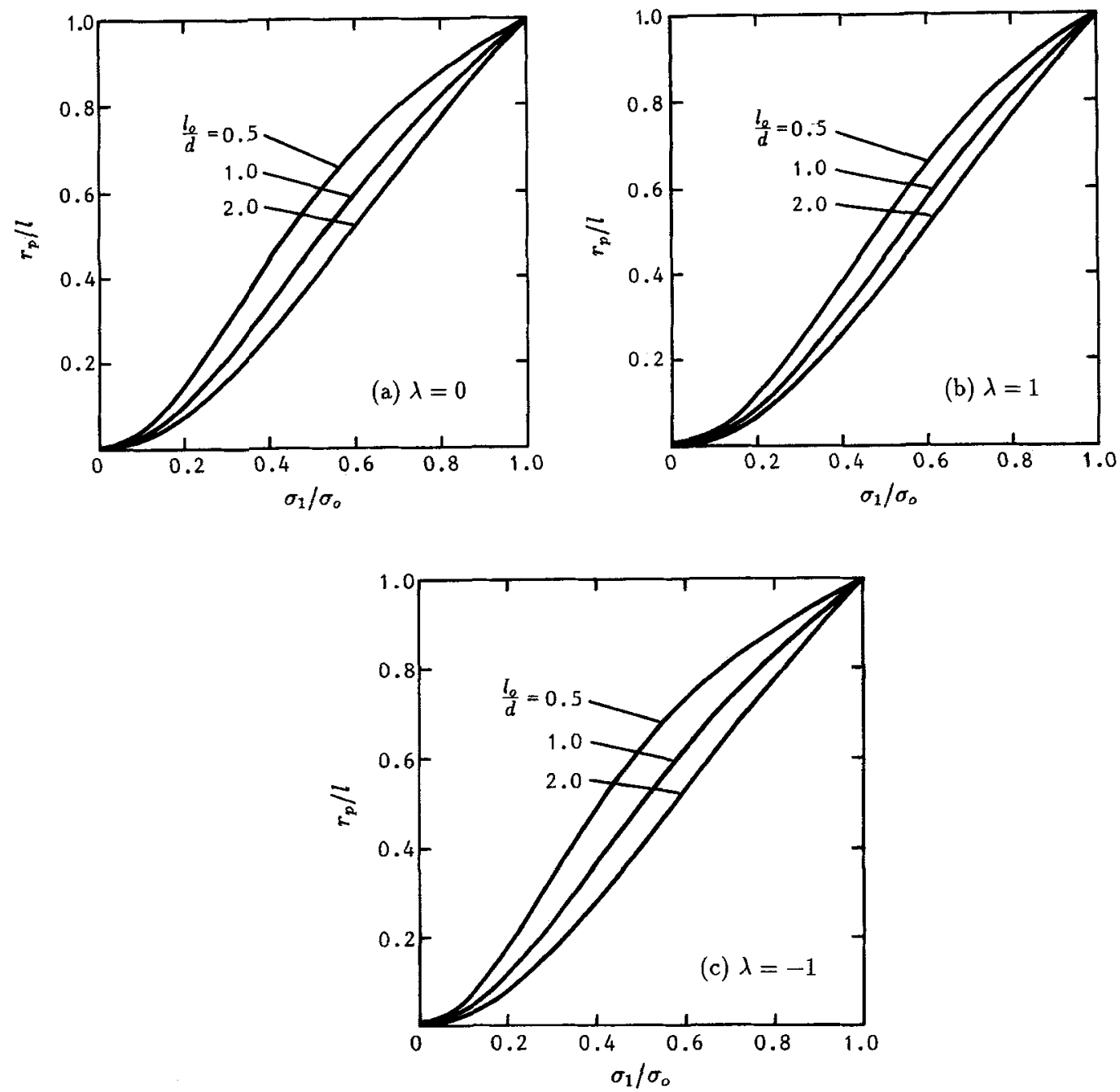

Fig. 9. Plastic zone sizes for cracks emanating from a circular notch at three biaxialities.

with $F_{0}, F_{1}$ and $F_{-1}$ being provided by equations (106), (108) and (110). Figure 9 demonstrates the results for these three biaxialities in the form of plastic zone size, $r_{\mathrm{p}} / l$, as a function of $\sigma_{1} / \sigma_{0}$ and $l_{0} / d$. The comparison of the present results with available numerical ones given in Table 1 clearly shows that the values calculated from equation (122) are in good agreement with those from Rich and Roberts [13] who regarded the yield stress $\sigma_{\mathrm{ys}}$ as $\sigma_{0}$ for the uniaxial tension. The difference, for most of the data pairs tabulated, in comparison with the present work is about or less than $1 \%$. It is worth noting that the cohesive stress $\sigma_{0}$ depends not only on the yield stress $\sigma_{\mathrm{ys}}$ but also on the $T$ stress that may prevail parallel to the mode I crack plane as a component of $\sigma_{x x}$ and is a function of stress biaxialities [14].

The same technique can be used to obtain similar results for cracks originating from an elliptical notch in an infinite plate subjected to remote biaxial loading. 
Table 1. Values of $\sigma_{1} / \sigma_{0}$ as a function of $r_{\mathrm{p}} / l$ and $l_{0} / d$ for $\lambda=0$

\begin{tabular}{clllllllll}
\hline$l_{0}$ & $\frac{r_{\mathrm{p}}}{d}$ & & & & & & & & \\
\hline & 0.1 & 0.2 & 0.3 & 0.4 & 0.5 & 0.6 & 0.7 & 0.8 & \\
\hline 0.5 & 0.162 & 0.239 & 0.306 & 0.372 & 0.441 & 0.515 & 0.601 & 0.703 & Eq. (122) \\
& 0.158 & 0.239 & 0.306 & 0.366 & 0.428 & 0.514 & 0.595 & 0.695 & Ref. [13] \\
1.0 & 0.199 & 0.292 & 0.371 & 0.446 & 0.522 & 0.600 & 0.684 & 0.777 & Eq. (122) \\
& 0.205 & 0.295 & 0.373 & 0.446 & 0.517 & 0.600 & 0.686 & 0.777 & Ref. [13] \\
2.0 & 0.232 & 0.337 & 0.429 & 0.506 & 0.583 & 0.661 & 0.740 & 0.822 & Eq. (122) \\
& 0.223 & 0.337 & 0.425 & 0.512 & 0.587 & 0.669 & 0.737 & 0.822 & Ref. [13] \\
\hline
\end{tabular}

\section{CONCLUSIONS}

A conformal mapping technique has been applied to the problem of an infinite plate containing a central lip-shaped notch subjected to biaxial loading at remote boundary and also for a uniform surface pressure on notch boundary profile. The derived complex stress functions can be used to obtain the stress distribution ahead of the notch and the stress intensity factors at the notch tip. The analytical $K$-expressions can be applied to a circular or an elliptical notch containing two symmetrical cracks, when the ratio of crack length to notch radius is in excess of a specified extent. Based on the present solutions, an analytical formula characterizing the plastic zone sizes for cracks emanating from a circular notch is obtained. These are of engineering importance and assist analytical derivations required for fatigue and failure analyses.

Acknowledgement - One of the authors (YH) wishes to thank the Royal Society for arranging his visit to the University of Sheffield and the award of a Royal Fellowship.

\section{REFERENCES}

1. O. L. Bowie (1956) Analysis of an infinite plate containing radial cracks originating from the boundary of an internal circular hole. J. Math. Phys. 35, 60-71.

2. J. C. Newman (1971) Am improved method of collocation for the stress analysis of cracked plates with various shaped boundaries. NASA TN D-6376.

3. M. Isida (1971) Effect of width and length on stress intensity factors of internally cracked plates under various boundary conditions. Int. J. Fract. Mech. 7, 301-316.

4. H. Nisitani (1978) Solutions of notch problems by body force method. Stress Analysis of Notch Problems (Edited by G. C. Sih), pp. 1-68. Noordhoff, Groningen.

5. J. Schijve (1982) The stress intensity factor of small cracks at notches. Fatigue Engng Mater. Struct. 5, $77-90$.

6. S. T. Xiao, M. W. Brown and K. J. Miller (1985) Stress intensity factors for cracks in notched finite plates subjected to biaxial loading. Fatigue Fract. Engng Mater. Struct. 8, 349-372.

7. D. P. Rooke, F. I. Baratta and D. J. Cartwright (1981) Simple methods of determining stress intensity factors. Engng Fract. Mech. 14, 397-426.

8. R. A. Smith and K. J. Miller (1977) Fatigue cracks at notches. Int. J. Mech. Sci. 19, 11-22.

9. N. I. Muskhelishvili (1953) Some Basic Problems of the Mathematical Theory of Elasticity, 3rd edn. Noordhoff, Groningen.

10. P. C. Paris and G. C. Sih (1965) Stress analysis of cracks. Fractire Toughness Testing and Its Applications. ASTM STP 381, pp. 30-83.

11. D. S. Dugdale (1960) Yielding of steel sheets containing slits. J. Mech. Phys. Solids 8, 100-104.

12. H. F. Bueckner (1973) Field singularities and related integral expressions. Methods of Analysis and Solutions of Crack Problems (Edited by G. C. Sih), pp. 239-314. Noordhoff, Groningen. 
13. T. Rich and R. Roberts (1968) Plastic enclave sizes for internal cracks emanating from circular cavities within elastic plates. Engng Fract. Mech. 1, 167-173.

14. M. W. Brown and K. J. Miller (1985) Mode I fatigue crack growth under biaxial stress at room and elevated temperature. Multiaxial Fatigue (Edited by K. J. Miller and M. W. Brown), ASTM-STP 853, pp. $135-152$. 